ICM 2002 · Vol. I · 449474

\title{
Galois Representations*
}

\author{
R. Taylor ${ }^{\dagger}$
}

\begin{abstract}
In the first part of this paper we try to explain to a general mathematical audience some of the remarkable web of conjectures linking representations of Galois groups with algebraic geometry, complex analysis and discrete subgroups of Lie groups. In the second part we briefly review some limited recent progress on these conjectures.
\end{abstract}

2000 Mathematics Subject Classification: 11F80.

Keywords and Phrases: Galois representations, L-function, Automorphic forms.

\section{Introduction}

The organisers requested a talk which would both be a colloquium style talk understandable to a wide spectrum of mathematicians and one which would survey the recent developments in the subject. I have found it hard to meet both desiderata, and have opted to concentrate on the former. Thus the first three sections of this paper contain a simple presentation of a web of deep conjectures connecting Galois representations to algebraic geometry, complex analysis and discrete subgroups of Lie groups. This will be of no interest to the specialist. My hope is that the result is not too banal and that it will give the non-specialist some idea of what motivates work in this area. I should stress that nothing I write here is original. In the final section I briefly review some of what is known about these conjectures and very briefly mention some of the available techniques. I also mention two questions which lie outside the topic we are discussing, but which would have important implications for it. Maybe someone can make progress on them?

Due to lack of space much of this article is too abbreviated. A somewhat expanded version is available on my website www. math.harvard.edu/ ${ }^{\sim}$ rtaylor and will hopefully be published elsewhere.

\footnotetext{
${ }^{*}$ The work on this article was partially supported by NSF Grant DMS-9702885.

†Department of Mathematics, Harvard University, 1 Oxford St., Cambridge, MA 02138, USA. E-mail: rtaylor@math.harvard.edu
} 


\section{Galois representations}

We will let $\mathbb{Q}$ denote the field of rational numbers and $\overline{\mathbb{Q}}$ denote the field of algebraic numbers, the algebraic closure of $\mathbb{Q}$. We will also let $G_{\mathbb{Q}}$ denote the group of automorphisms of $\overline{\mathbb{Q}}$, that is $\operatorname{Gal}(\overline{\mathbb{Q}} / \mathbb{Q})$, the absolute Galois group of $\mathbb{Q}$. Although it is not the simplest it is arguably the most natural Galois group to study. An important technical point is that $G_{\mathbb{Q}}$ is naturally a (profinite) topological group, a basis of open neighbourhoods of the identity being given by the subgroups $\operatorname{Gal}(\overline{\mathbb{Q}} / K)$ as $K$ runs over subextensions of $\overline{\mathbb{Q}} / \mathbb{Q}$ which are finite over $\mathbb{Q}$.

To my mind the Galois theory of $\mathbb{Q}$ is most interesting when one looks not only at $G_{\mathbb{Q}}$ as an abstract (topological) group, but as a group with certain additional structures associated to the prime numbers. I will now briefly describe these structures.

For each prime number $p$ we may define an absolute value ||$_{p}$ on $\mathbb{Q}$ by setting

$$
|\alpha|_{p}=p^{-r}
$$

if $\alpha=p^{r} a / b$ with $a$ and $b$ integers coprime to $p$. If we complete $\mathbb{Q}$ with respect to this absolute value we obtain the field of $p$-adic numbers $\mathbb{Q}_{p}$, a totally disconnected, locally compact topological field. We will write $G_{\mathbb{Q}_{p}}$ for its absolute Galois group, $\operatorname{Gal}\left(\overline{\mathbb{Q}}_{p} / \mathbb{Q}_{p}\right)$. The absolute value ||$_{p}$ has a unique extension to an absolute value on $\overline{\mathbb{Q}}_{p}$ and $G_{\mathbb{Q}_{p}}$ is identified with the group of automorphisms of $\overline{\mathbb{Q}}_{p}$ which preserve ||$_{p}$, or equivalently the group of continuous automorphisms of $\overline{\mathbb{Q}}_{p}$. For each embedding $\overline{\mathbb{Q}} \hookrightarrow \overline{\mathbb{Q}}_{p}$ we obtain a closed embedding $G_{\mathbb{Q}_{p}} \hookrightarrow G_{\mathbb{Q}}$ and as the embedding $\overline{\mathbb{Q}} \hookrightarrow \overline{\mathbb{Q}}_{p}$ varies we obtain a conjugacy class of closed embeddings $G_{\mathbb{Q}_{p}} \hookrightarrow G_{\mathbb{Q}}$. Slightly abusively, we shall consider $G_{\mathbb{Q}_{p}}$ a closed subgroup of $G_{\mathbb{Q}}$, suppressing the fact that the embedding is only determined up to conjugacy.

This can be compared with the situation 'at infinity'. Let $\left.\right|_{\infty}$ denote the usual Archimedean absolute value on $\mathbb{Q}$. The completion of $\mathbb{Q}$ with respect to $\left.\right|_{\infty}$ is the field of real numbers $\mathbb{R}$ and its algebraic closure is $\mathbb{C}$ the field of complex numbers. Each embedding $\overline{\mathbb{Q}} \hookrightarrow \mathbb{C}$ gives rise to a closed embedding

$$
\{1, c\}=G_{\mathbb{R}}=\operatorname{Gal}(\mathbb{C} / \mathbb{R}) \hookrightarrow G_{\mathbb{Q}} .
$$

As the embedding $\overline{\mathbb{Q}} \hookrightarrow \mathbb{C}$ varies one obtains a conjugacy class of elements $c \in G_{\mathbb{Q}}$ of order 2 , which we refer to as complex conjugations.

There are however many important differences between the case of finite places (i.e. primes) and the infinite place ||$_{\infty}$. For instance $\overline{\mathbb{Q}}_{p} / \mathbb{Q}_{p}$ is an infinite extension and $\overline{\mathbb{Q}}_{p}$ is not complete. We will denote its completion by $\mathbb{C}_{p}$. The Galois group $G_{\mathbb{Q}_{p}}$ acts on $\mathbb{C}_{p}$ and is in fact the group of continuous automorphisms of $\mathbb{C}_{p}$.

The elements of $\mathbb{Q}_{p}$ (resp. $\overline{\mathbb{Q}}_{p}$ ) with absolute value less than or equal to 1 , form a closed subring $\mathbb{Z}_{p}$ (resp. $\mathcal{O}_{\overline{\mathbb{Q}}_{p}}$ ). These rings are local with maximal ideals $p \mathbb{Z}_{p}$ (resp. $\mathfrak{m}_{\overline{\mathbb{Q}}_{p}}$ ) consisting of the elements with absolute value strictly less than 1. The field $\mathcal{O}_{\overline{\mathbb{Q}}_{p}} / \mathfrak{m}_{\overline{\mathbb{Q}}_{p}}$ is an algebraic closure of the finite field with $p$ elements $\mathbb{F}_{p}=\mathbb{Z}_{p} / p \mathbb{Z}_{p}$, and we will denote it by $\overline{\mathbb{F}}_{p}$. Thus we obtain a continuous map

$$
G_{\mathbb{Q}_{p}} \longrightarrow G_{\mathbb{F}_{p}}
$$


which is surjective. Its kernel is called the inertia subgroup of $G_{\mathbb{Q}_{p}}$ and is denoted by $I_{\mathbb{Q}_{p}}$. The group $G_{\mathbb{F}_{p}}$ is procyclic and has a canonical generator called the (geometric) Frobenius element and defined by

$$
\operatorname{Frob}_{p}^{-1}(x)=x^{p}
$$

In many circumstances it is technically convenient to replace $G_{\mathbb{Q}_{p}}$ by a dense subgroup $W_{\mathbb{Q}_{p}}$, which is referred to as the Weil group of $\mathbb{Q}_{p}$ and which is defined as the subgroup of $\sigma \in G_{\mathbb{Q}_{p}}$ such that $\sigma$ maps to

$$
\operatorname{Frob}_{p}^{\mathbb{Z}} \subset G_{\mathbb{F}_{p}} .
$$

We endow $W_{\mathbb{Q}_{p}}$ with a topology by decreeing that $I_{\mathbb{Q}_{p}}$ with its usual topology should be an open subgroup of $W_{\mathbb{Q}_{p}}$.

We will take a moment to describe some of the finer structure of $I_{\mathbb{Q}_{p}}$ which we will need for technical purposes later. First of all there is a (not quite canonical) continuous surjection

$$
I_{\mathbb{Q}_{p}} \rightarrow \prod_{l \neq p} \mathbb{Z}_{l}
$$

such that

$$
t\left(\operatorname{Frob}_{p} \sigma \operatorname{Frob}_{p}^{-1}\right)=p^{-1} t(\sigma)
$$

for all $\sigma \in I_{\mathbb{Q}_{p}}$. The kernel of $t$ is a pro-p-group called the wild inertia group. The fixed field $\overline{\mathbb{Q}}_{p}^{\text {ker } t}$ is obtained by adjoining $\sqrt[n]{p}$ to $\overline{\mathbb{Q}}_{p}^{I_{\mathbb{Q}_{p}}}$ for all $n$ coprime to $p$ and

$$
\sigma \sqrt[n]{p}=\zeta_{n}^{t(\sigma)} \sqrt[n]{p}
$$

for some primitive $n^{\text {th }}$-root of unity $\zeta_{n}$ (independent of $\sigma$, but dependent on $t$ ).

In my opinion the most interesting question about $G_{\mathbb{Q}}$ is to describe it together with the distinguished subgroups $G_{\mathbb{R}}, G_{\mathbb{Q}_{p}}, I_{\mathbb{Q}_{p}}$ and the distinguished elements $\operatorname{Frob}_{p} \in G_{\mathbb{Q}_{p}} / I_{\mathbb{Q}_{p}}$.

I want to focus here on attempts to describe $G_{\mathbb{Q}}$ via its representations. Perhaps the most obvious representations to consider are those representations

$$
G_{\mathbb{Q}} \longrightarrow G L_{n}(\mathbb{C})
$$

with open kernel, and these so called Artin representations are already very interesting. However one obtains a richer theory if one considers representations

$$
G_{\mathbb{Q}} \longrightarrow G L_{n}\left(\overline{\mathbb{Q}}_{l}\right)
$$

which are continuous with respect to the $l$-adic topology on $G L_{n}\left(\overline{\mathbb{Q}}_{l}\right)$. We refer to these as $l$-adic representations.

One justification for considering $l$-adic representations is that they arise naturally from geometry. Here are some examples of $l$-adic representations.

1. A choice of embeddings $\overline{\mathbb{Q}} \hookrightarrow \mathbb{C}$ and $\overline{\mathbb{Q}} \hookrightarrow \overline{\mathbb{Q}}_{l}$ establishes a bijection between isomorphism classes of Artin representations and isomorphism classes of $l$-adic representations with open kernel. Thus Artin representations are a special case of $l$-adic representations: those with finite image. 
2. There is a unique character

$$
\chi_{l}: G_{\mathbb{Q}} \longrightarrow \mathbb{Z}_{l}^{\times} \subset \overline{\mathbb{Q}}_{l}^{\times}
$$

such that

$$
\sigma \zeta=\zeta^{\chi_{l}(\sigma)}
$$

for all $l$-power roots of unity $\zeta$. This is called the $l$-adic cyclotomic character.

3. If $X / \mathbb{Q}$ is a smooth projective variety (and we choose an embedding $\overline{\mathbb{Q}} \subset \mathbb{C}$ )

then the natural action of $G_{\mathbb{Q}}$ on the cohomology

$$
H^{i}\left(X(\mathbb{C}), \overline{\mathbb{Q}}_{l}\right) \cong H_{\mathrm{et}}^{i}\left(X \times_{\mathbb{Q}} \overline{\mathbb{Q}}, \overline{\mathbb{Q}}_{l}\right)
$$

is an $l$-adic representation. For instance if $E / \mathbb{Q}$ is an elliptic curve then we have the concrete description

$$
H_{\text {et }}^{1}\left(E \times_{\mathbb{Q}} \overline{\mathbb{Q}}, \overline{\mathbb{Q}}_{l}\right) \cong \operatorname{Hom}_{\mathbb{Z}_{l}}\left(\lim _{\leftarrow r} E\left[l^{r}\right](\overline{\mathbb{Q}}), \overline{\mathbb{Q}}_{l}\right) \cong \overline{\mathbb{Q}}_{l}^{2},
$$

where $E\left[l^{r}\right]$ denotes the $l^{r}$-torsion points on $E$. We will write $H^{i}\left(X(\mathbb{C}), \overline{\mathbb{Q}}_{l}(j)\right)$ for the twist

$$
H^{i}\left(X(\mathbb{C}), \overline{\mathbb{Q}}_{l}\right) \otimes \chi_{l}^{j}
$$

Before discussing $l$-adic representations of $G_{\mathbb{Q}}$ further, let us take a moment to look at $l$-adic representations of $G_{\mathbb{Q}_{p}}$. The cases $l \neq p$ and $l=p$ are very different. Consider first the much easier case $l \neq p$. Here $l$-adic representations of $G_{\mathbb{Q}_{p}}$ are not much different from representations of $W_{\mathbb{Q}_{p}}$ with open kernel. More precisely define a $W D$-representation of $W_{\mathbb{Q}_{p}}$ over a field $E$ to be a pair

$$
r: W_{\mathbb{Q}_{p}} \longrightarrow G L(V)
$$

and

$$
N \in \operatorname{End}(V),
$$

where $V$ is a finite dimensional $E$-vector space, $r$ is a representation with open kernel and $N$ is a nilpotent endomorphism which satisfies

$$
r(\phi) N r\left(\phi^{-1}\right)=p^{-1} N
$$

for every lift $\phi \in W_{\mathbb{Q}_{p}}$ of $\operatorname{Frob}_{p}$. The key point here is that there is no reference to a topology on $E$, indeed no assumption that $E$ is a topological field. Given $r$ there are up to isomorphism only finitely many choices for the pair $(r, N)$ and these can be explicitly listed without difficulty. A WD-representation $(r, N)$ is called unramified if $N=0$ and $r\left(I_{\mathbb{Q}_{p}}\right)=\{1\}$. It is called Frobenius semi-simple if $r$ is semi-simple. Any WD-representation $(r, N)$ has a canonical Frobenius semi-simplification $(r, N)^{\mathrm{ss}}$ (see [Tat]). In the case that $E=\overline{\mathbb{Q}}_{l}$ we call $(r, N) l$-integral if all the eigenvalues of $r(\phi)$ have absolute value 1 . This is independent of the choice of Frobenius lift $\phi$.

If $l \neq p$, then there is an equivalence of categories between $l$-integral WDrepresentations of $W_{\mathbb{Q}_{p}}$ over $\overline{\mathbb{Q}}_{l}$ and $l$-adic representations of $G_{\mathbb{Q}_{p}}$. To describe it choose a Frobenius lift $\phi \in W_{\mathbb{Q}_{p}}$ and a surjection $t_{l}: I_{\mathbb{Q}_{p}} \rightarrow \mathbb{Z}_{l}$. Up to natural 
isomorphism the equivalence does not depend on these choices. We associate to an $l$-integral WD-representation $(r, N)$ the unique $l$-adic representation sending

$$
\phi^{n} \sigma \longmapsto r\left(\phi^{n} \sigma\right) \exp \left(t_{l}(\sigma) N\right)
$$

for all $n \in \mathbb{Z}$ and $\sigma \in I_{\mathbb{Q}_{p}}$. The key point is Grothendieck's observation that for $l \neq p$ any $l$-adic representation of $G_{\mathbb{Q}_{p}}$ must be trivial on some open subgroup of the wild inertia group. We will write $\mathrm{WD}_{p}(R)$ for the WD-representation associated to an $l$-adic representation $R$. Note that $\mathrm{WD}_{p}(R)$ is unramified if and only if $R\left(I_{p}\right)=\{1\}$. In this case we call $R$ unramified.

The case $l=p$ is much more complicated because there are many more $p$ adic representations of $G_{\mathbb{Q}_{p}}$. These have been extensively studied by Fontaine and his co-workers. They single out certain $p$-adic representations which they call de Rham representations. I will not recall the somewhat involved definition here (see however [Fo2] and [Fo3]), but note that 'most' $p$-adic representations of $G_{\mathbb{Q}_{p}}$ are not de Rham. To any de Rham representation $R$ of $G_{\mathbb{Q}_{p}}$ on a $\overline{\mathbb{Q}}_{p}$-vector space $V$ they associate the following.

1. A WD-representation $\mathrm{WD}_{p}(R)$ of $W_{\mathbb{Q}_{p}}$ over $\overline{\mathbb{Q}}_{p}$ (see [Berg] and [Fo4]).

2. A multiset $\mathrm{HT}(R)$ of $\operatorname{dim} V$ integers, called the Hodge-Tate numbers of $R$. The multiplicity of $i$ in $\operatorname{HT}(R)$ is

$$
\operatorname{dim}_{\overline{\mathbb{Q}}_{p}}\left(V \otimes_{\mathbb{Q}_{p}} \mathbb{C}_{p}(i)\right)^{G_{\mathbb{Q}_{p}}},
$$

where $\mathbb{C}_{p}(i)$ denotes $\mathbb{C}_{p}$ with $G_{\mathbb{Q}_{p}}$-action $\chi_{p}(\sigma)^{i}$ times the usual (Galois) action on $\mathbb{C}_{p}$.

We now return to the global situation (i.e. to the study of $G_{\mathbb{Q}}$ ). The $l$-adic representations of $G_{\mathbb{Q}}$ that arise 'in nature', by which I mean 'from geometry', have a number of very special properties which I will now list. Let $R: G_{\mathbb{Q}} \longrightarrow G L(V)$ be a subquotient of $H^{i}\left(X(\mathbb{C}), \overline{\mathbb{Q}}_{l}(j)\right)$ for some smooth projective variety $X / \mathbb{Q}$ and some integers $i \geq 0$ and $j$.

1. (Grothendieck) The representation $R$ is unramified at all but finitely many primes $p$.

2. (Fontaine, Messing, Faltings, Kato, Tsuji, de Jong, see e.g. [I], [Bert]) The representation $R$ is de Rham in the sense that its restriction to $G_{\mathbb{Q}_{l}}$ is de Rham.

3. (Deligne, $[\mathrm{D}]$ ) The representation $R$ is pure of weight $w=i-2 j$ in the following sense. There is a finite set of primes $S$, such that for $p \notin S$, the representation $R$ is unramified at $p$ and for every eigenvalue $\alpha$ of $R\left(\right.$ Frob $\left._{p}\right)$ and every embedding $\iota: \overline{\mathbb{Q}}_{l} \hookrightarrow \mathbb{C}$

$$
|\iota \alpha|_{\infty}^{2}=p^{w}
$$

In particular $\alpha$ is algebraic (i.e. $\alpha \in \overline{\mathbb{Q}}$ ).

An amazing conjecture of Fontaine and Mazur (see [Fo1] and [FM]) asserts that any irreducible $l$-adic representation of $G_{\mathbb{Q}}$ satisfying the first two of these properties arises from geometry in the above sense and so in particular also satisfies the third property. 
Conjecture 1.1 (Fontaine-Mazur) Suppose that

$$
R: G_{\mathbb{Q}} \longrightarrow G L(V)
$$

is an irreducible l-adic representation which is unramified at all but finitely many primes and with $\left.R\right|_{G_{\mathbb{Q}_{I}}}$ de Rham. Then there is a smooth projective variety $X / \mathbb{Q}$ and integers $i \geq 0$ and $j$ such that $V$ is a subquotient of $H^{i}\left(X(\mathbb{C}), \overline{\mathbb{Q}}_{l}(j)\right)$. In particular $R$ is pure of some weight $w \in \mathbb{Z}$.

We will discuss the evidence for this conjecture later. We will call an $l$-adic representation satisfying the conclusion of this conjecture geometric.

Algebraic geometers have formulated some very precise conjectures about the action of $G_{\mathbb{Q}}$ on the cohomology of varieties. We don't have the space here to discuss these in general, but we will formulate, in an as algebraic a way as possible, some of their conjectures.

Conjecture 1.2 (Tate) Suppose that $X / \mathbb{Q}$ is a smooth projective variety. Then there is a decomposition

$$
H^{i}(X(\mathbb{C}), \overline{\mathbb{Q}})=\bigoplus_{j} M_{j}
$$

with the following properties.

1. For each prime $l$ and for each embedding $\iota: \overline{\mathbb{Q}} \hookrightarrow \overline{\mathbb{Q}}_{l}, M_{j} \otimes_{\overline{\mathbb{Q}}, \iota} \overline{\mathbb{Q}}_{l}$ is an irreducible subrepresentation of $H^{i}\left(X(\mathbb{C}), \overline{\mathbb{Q}}_{l}\right)$.

2. For all indices $j$ and for all primes $p$ there is a WD-representation $\mathrm{WD}_{p}\left(M_{j}\right)$ of $W_{\mathbb{Q}_{p}}$ over $\overline{\mathbb{Q}}$ such that

$$
\mathrm{WD}_{p}\left(M_{j}\right) \otimes_{\overline{\mathbb{Q}}, \iota} \overline{\mathbb{Q}}_{l} \cong \mathrm{WD}_{p}\left(M_{j} \otimes_{\overline{\mathbb{Q}}, \iota} \overline{\mathbb{Q}}_{l}\right)
$$

for all primes $l$ and all embeddings $\iota: \overline{\mathbb{Q}} \hookrightarrow \overline{\mathbb{Q}}_{l}$.

3. There is a multiset of integers $\mathrm{HT}\left(M_{j}\right)$ such that

(a) for all primes $l$ and all embeddings $\iota: \overline{\mathbb{Q}} \hookrightarrow \overline{\mathbb{Q}}_{l}$

$$
\operatorname{HT}\left(M_{j} \otimes_{\overline{\mathbb{Q}}, \iota} \overline{\mathbb{Q}}_{l}\right)=H T\left(M_{j}\right)
$$

(b) and for all $\iota: \overline{\mathbb{Q}} \hookrightarrow \mathbb{C}$

$$
\operatorname{dim}_{\mathbb{C}}\left(\left(M_{j} \otimes_{\overline{\mathbb{Q}}, \iota} \mathbb{C}\right) \cap H^{a, i-a}(X(\mathbb{C}), \mathbb{C})\right)
$$

is the multiplicity of a in $H T\left(M_{j}\right)$.

If one considers the whole of $H^{i}(X(\mathbb{C}), \overline{\mathbb{Q}})$ rather than its pieces $M_{j}$, then part 2. is known to hold up to Frobenius semisimplification for all but finitely many $p$ and part 3. is known to hold (see [1] ). It follows from a theorem of Faltings Fa. that the whole conjecture is true for $H^{1}$ of an abelian variety. The putative constituents $M_{j}$ are one incarnation of what people call 'pure motives'.

If one believes conjectures 1.1 and 1.2 then 'geometric' $l$-adic representations should come in compatible families as $l$ varies. There are many ways to make precise the notion of such a compatible family. Here is one. 
By a weakly compatible system of $l$-adic representations $\mathcal{R}=\left\{R_{l, \ell}\right\}$ we shall mean a collection of semi-simple $l$-adic representations

$$
R_{l, \iota}: G_{\mathbb{Q}} \longrightarrow G L\left(V \otimes_{\overline{\mathbb{Q}}, \iota} \overline{\mathbb{Q}}_{l}\right),
$$

one for each pair $(l, \iota)$, where $l$ is a prime and $\iota: \overline{\mathbb{Q}} \hookrightarrow \overline{\mathbb{Q}}_{l}$, which satisfy the following conditions.

- There is a multiset of integers $\operatorname{HT}(\mathcal{R})$ such that for each prime $l$ and each embedding $\iota: \overline{\mathbb{Q}} \hookrightarrow \overline{\mathbb{Q}}_{l}$ the restriction $\left.R_{l, \iota}\right|_{G_{\mathbb{l}_{l}}}$ is de Rham and $\operatorname{HT}\left(\left.R_{l, \iota}\right|_{G_{\mathbb{Q}_{l}}}\right)=$ $\operatorname{HT}(\mathcal{R})$.

- There is a finite set of primes $S$ such that if $p \notin S$ then $\operatorname{WD}_{p}\left(R_{l, \iota}\right)$ is unramified for all $l$ and $\iota$.

- For all but finitely many primes $p$ there is a Frobenius semi-simple WDrepresentation $\mathrm{WD}_{p}(\mathcal{R})$ over $\overline{\mathbb{Q}}$ such that for all primes $l \neq p$ and for all $\iota$ we have

$$
\mathrm{WD}_{p}\left(R_{l, \iota}\right)^{\mathrm{ss}} \sim \mathrm{WD}_{p}(\mathcal{R}) .
$$

We make the following subsidiary definitions.

- We call $\mathcal{R}$ strongly compatible if the last condition (the existence of $\mathrm{WD}_{p}(\mathcal{R})$ ) holds for all primes $p$.

- We call $\mathcal{R}$ irreducible if each $R_{l, \iota}$ is irreducible.

- We call $\mathcal{R}$ pure of weight $w \in \mathbb{Z}$, if for all but finitely many $p$ and for all eigenvalues $\alpha$ of $r_{p}\left(\operatorname{Frob}_{p}\right)$, where $\mathrm{WD}_{p}(\mathcal{R})=\left(r_{p}, N_{p}\right)$, we have

$$
|\iota \alpha|_{\infty}^{2}=p^{w}
$$

for all embeddings $\iota: \overline{\mathbb{Q}} \hookrightarrow \mathbb{C}$.

- We call $\mathcal{R}$ geometric if there is a smooth projective variety $X / \mathbb{Q}$ and integers $i \geq 0$ and $j$ and a subspace

$$
W \subset H^{i}(X(\mathbb{C}), \overline{\mathbb{Q}})
$$

such that for all $l$ and $\iota, W \otimes_{\overline{\mathbb{Q}}, \iota} \overline{\mathbb{Q}}_{l}$ is $G_{\mathbb{Q}}$ invariant and realises $R_{l, \iota}$.

Conjectures 1.1 and 1.2 lead one to make the following conjecture.

Conjecture 1.3 1. If $R: G_{\mathbb{Q}} \rightarrow G L_{n}\left(\overline{\mathbb{Q}}_{l}\right)$ is a continuous semi-simple de Rham representation unramified at all but finitely many primes then $R$ is part of a weakly compatible system.

2. Any weakly compatible system is strongly compatible.

3. Any irreducible weakly compatible system $\mathcal{R}$ is geometric and pure of weight $(2 / \operatorname{dim} \mathcal{R}) \sum_{h \in \mathrm{HT}(\mathcal{R})} h$.

A famous theorem of Cebotarev asserts that if $K / \mathbb{Q}$ is any Galois extension in which all but finitely many primes are unramified (i.e. for all but finitely many primes $p$ the image of $I_{\mathbb{Q}_{p}}$ in $\operatorname{Gal}(K / \mathbb{Q})$ is trivial) then the Frobenius elements at unramified primes $\operatorname{Frob}_{p} \in \operatorname{Gal}(K / \mathbb{Q})$ are dense in $\operatorname{Gal}(K / \mathbb{Q})$. It follows that an 
irreducible weakly compatible system $\mathcal{R}$ is uniquely determined by $\mathrm{WD}_{p}(\mathcal{R})$ for all but finitely many $p$ and hence by one $R_{l, \iota}$.

Conjectures 1.1 and 1.3 are known for one dimensional representations, in which case they have purely algebraic proofs based on class field theory (see $[\mathrm{Se}]$ ). Otherwise only fragmentary cases have been proved, where amazingly the arguments are extremely indirect involving sophisticated analysis and geometry. We will come back to this later.

\section{2. $\quad L$-functions}

$L$-functions are certain Dirichlet series

$$
\sum_{n=1}^{\infty} a_{n} / n^{s}
$$

which play an important role in number theory. A full discussion of the role of $L$ functions in number theory is beyond the scope of this talk. The simplest example of an $L$-function is the Riemann zeta function

$$
\zeta(s)=\sum_{n=1}^{\infty} 1 / n^{s} .
$$

It converges to a holomorphic function in the half plane $\operatorname{Re} s>1$ and in this region of convergence it can also be expressed as a convergent infinite product over the prime numbers

$$
\zeta(s)=\prod_{p}\left(1-1 / p^{s}\right)^{-1} .
$$

This is called an Euler product and the individual factors are called Euler factors. Lying deeper is the fact that $\zeta(s)$ has meromorphic continuation to the whole complex plane, with only one pole: a simple pole at $s=1$. Moreover if we set

$$
Z(s)=\pi^{-s / 2} \Gamma(s / 2) \zeta(s)
$$

then $Z$ satisfies the functional equation

$$
Z(1-s)=Z(s)
$$

Encoded in the Riemann zeta function is lots of deep arithmetic information. For instance the location of the zeros of $\zeta(s)$ is intimately connected with the distribution of prime numbers. Moreover its special values at negative integers (where it is only defined by analytic continuation) turn out to be rational numbers encoding deep arithmetic information about the cyclotomic fields $\mathbb{Q}\left(e^{2 \pi \sqrt{-1} / p}\right)$.

Another celebrated example is the $L$-function of an elliptic curve $E$ :

$$
y^{2}=x^{3}+a x+b .
$$


In this case the $L$-function is defined as an Euler product (converging in $\operatorname{Re} s>3 / 2$ )

$$
L(E, s)=\prod_{p} L_{p}\left(E, p^{-s}\right),
$$

where $L_{p}(E, X)$ is a rational function, and for all but finitely many $p$

$$
L_{p}(E, X)=\left(1-a_{p}(E) X+p X^{2}\right)^{-1}
$$

with $p-a_{p}(E)$ being the number of solutions to the congruence

$$
y^{2} \equiv x^{3}+a x+b \bmod p
$$

in $\mathbb{F}_{p}^{2}$. It has recently been proved BCDT that $L(E, s)$ can be continued to an entire function, which satisfies a functional equation

$$
(2 \pi)^{-s} \Gamma(s) L(E, s)= \pm N(E)^{1-s}(2 \pi)^{s-2} \Gamma(2-s) L(E, 2-s)
$$

for some explicit positive integer $N(E)$. A remarkable conjecture of Birch and Swinnerton-Dyer [BSD] predicts that $y^{2}=x^{3}+a x+b$ has infinitely many rational solutions if and only if $L(E, 1)=0$. Again we point out that it is the behaviour of the $L$-function at a point where it is only defined by analytic continuation, which is governing the arithmetic of $E$. This conjecture has been proved (see [Ko] ]) when $L(E, s)$ has at most a simple zero at $s=1$.

One general setting in which one can define $L$-functions is $l$-adic representations. Let us look first at the local setting. If $(r, N)$ is a WD-representation of $W_{\mathbb{Q}_{p}}$ on an $E$-vector space $V$, where $E$ is an algebraically closed field of characteristic zero, we define a local L-factor

$$
L((r, N), X)=\left.\operatorname{det}\left(1-X \operatorname{Frob}_{p}\right)\right|_{V^{I_{\mathbb{Q}_{p}}, N=0}} ^{-1} \in E(X) .
$$

$\left(V^{I_{\mathbb{Q}_{p}}, N=0}\right.$ is the subspace of $V$ where $I_{\mathbb{Q}_{p}}$ acts trivially and $N=0$.) One can also associate to $(r, N)$ a conductor $f(r, N) \in \mathbb{Z}_{\geq 0}$, which measures how deeply into $I_{\mathbb{Q}_{p}}$ the WD-representation $(r, N)$ is nontrivial, and a local epsilon factor $\epsilon\left((r, N), \Psi_{p}\right) \in$ $E$, which also depends on the choice of a non-trivial character $\Psi_{p}: \mathbb{Q}_{p} \rightarrow E^{\times}$with open kernel. (See [Tat].)

If $R: G_{\mathbb{Q}} \rightarrow G L(V)$ is an $l$-adic representation of $G_{\mathbb{Q}}$ which is de Rham at $l$ and pure of some weight $w \in \mathbb{Z}$, and if $\iota: \overline{\mathbb{Q}}_{l} \hookrightarrow \mathbb{C}$ we will define an $L$-function

$$
L(\iota R, s)=\prod_{p} L\left(\iota \mathrm{WD}_{p}(R), p^{-s}\right),
$$

which will converge to a holomorphic function in $\operatorname{Re} s>1+w / 2$. For example

$$
L(1, s)=\zeta(s)
$$

and if $E / \mathbb{Q}$ is an elliptic curve then

$$
L\left(\iota H^{1}\left(E(\mathbb{C}), \overline{\mathbb{Q}}_{l}\right), s\right)=L(E, s)
$$


(for any $\iota$ ). Note the useful formulae

$$
L\left(\iota\left(R_{1} \oplus R_{2}\right), s\right)=L\left(\iota R_{1}, s\right) L\left(\iota R_{2}, s\right) \quad \text { and } \quad L\left(\iota\left(R \otimes \chi_{l}^{r}\right), s\right)=L(\iota R, s+r) .
$$

Also note that $L(\iota R, s)$ determines $L\left(\mathrm{WD}_{p}(R), X\right)$ for all $p$ and hence $\mathrm{WD}_{p}(R)^{\mathrm{ss}}$ for all but finitely many $p$. Hence by the Cebotarev density theorem $L(\iota R, s)$ determines $R$ (up to semisimplification).

Write $m_{i}^{R}$ for the multiplicity of an integer $i$ in $\operatorname{HT}(R)$ and, if $w / 2 \in \mathbb{Z}$, define $m_{w / 2, \pm}^{R} \in(1 / 2) \mathbb{Z}$ by:

$$
\begin{aligned}
& m_{w / 2,+}^{R}+m_{w / 2,-}^{R}=m_{w / 2}^{R} \\
& m_{w / 2,+}^{R}-m_{w / 2,-}^{R}=(-1)^{w / 2}\left(\operatorname{dim} V^{c=1}-\operatorname{dim} V^{c=-1}\right) .
\end{aligned}
$$

Assume that $m_{w / 2, \pm}^{R}$ are integers, i.e. that $m_{w / 2}^{R} \equiv \operatorname{dim} V \bmod 2$. Then we can define a $\Gamma$-factor, $\Gamma(R, s)$, which is a product of functions $\pi^{-(s+a) / 2} \Gamma((s+a) / 2)$ as $a$ runs over a set of integers depending only on the numbers $m_{i}^{R}$ and $m_{w / 2, \pm}^{R}$. We can also define an epsilon factor $\epsilon_{\infty}\left(R, \Psi_{\infty}\right) \in \mathbb{C}^{\times}$which again only depends on $m_{i}^{R}, m_{w / 2, \pm}^{R}$ and a non-trivial character $\Psi_{\infty}: \mathbb{R} \rightarrow \mathbb{C}^{\times}$. Set

$$
\Lambda(\iota R, s)=\Gamma(R, s) L(\iota R, s)
$$

and

$$
N(R)=\prod_{p} p^{f\left(\mathrm{WD}_{p}(R)\right)}
$$

(which makes sense as $f\left(\mathrm{WD}_{p}(R)\right)=0$ for all but finitely many $p$ ) and

$$
\epsilon(\iota R)=\epsilon_{\infty}\left(R, e^{2 \pi \sqrt{-1} x}\right) \prod_{p} \iota \epsilon\left(\mathrm{WD}_{p}(R), \Psi_{p}\right),
$$

where $\iota \Psi_{p}(x)=e^{-2 \pi \sqrt{-1} x}$.

The following conjecture is a combination of conjecture 1.1 and conjectures which have become standard.

Conjecture 2.1 Suppose that $R$ is an irreducible l-adic representation of $G_{\mathbb{Q}}$ which is de Rham and pure of weight $w \in \mathbb{Z}$. Then $m_{p}^{R}=m_{w-p}^{R}$ for all $p$, so that $m_{w / 2} \equiv \operatorname{dim} V \bmod 2$. Moreover the following should hold.

1. $L(\iota R, s)$ extends to an entire function, except for a single simple pole if $R=$ $\chi_{l}^{-w / 2}$.

2. $\Lambda(\iota R, s)$ is bounded in vertical strips $\sigma_{0} \leq \operatorname{Re} s \leq \sigma_{1}$.

3. $\Lambda(\iota R, s)=\epsilon(\iota R) N(R)^{-s} \Lambda\left(\iota R^{\vee}, 1-s\right)$.

It is tempting to believe that something like properties 1., 2. and 3. should characterise those Euler products which arise from $l$-adic representations. We will discuss a more precise conjecture along these lines in the next section. Why Galois representations should be the source of Euler products with good functional equations seems a complete mystery. 


\section{Automorphic forms}

Automorphic forms may be thought of as certain smooth functions on the quotient $G L_{n}(\mathbb{Z}) \backslash G L_{n}(\mathbb{R})$. We need several preliminaries before we can make a precise definition.

Let $\widehat{\mathbb{Z}}$ denote the profinite completion of $\mathbb{Z}$, i.e.

$$
\widehat{\mathbb{Z}}=\lim _{\leftarrow N} \mathbb{Z} / N \mathbb{Z}=\prod_{p} \mathbb{Z}_{p},
$$

a topological ring. Also let $\mathbb{A}^{\infty}$ denote the topological ring of finite adeles

$$
\mathbb{A}^{\infty}=\widehat{\mathbb{Z}} \otimes_{\mathbb{Z}} \mathbb{Q},
$$

where $\widehat{\mathbb{Z}}$ is an open subring with its usual topology. As an abstract ring, $\mathbb{A}^{\infty}$ is the subring of $\prod_{p} \mathbb{Q}_{p}$ consisting of elements $\left(x_{p}\right)$ with $x_{p} \in \mathbb{Z}_{p}$ for all but finitely many $p$. However the topology is not the subspace topology. We define the topological ring of adeles to be the product

$$
\mathbb{A}=\mathbb{A}^{\infty} \times \mathbb{R}
$$

Note that $\mathbb{Q}$ embeds diagonally as a discrete subring of $\mathbb{A}$ with compact quotient

$$
\mathbb{Q} \backslash \mathbb{A}=\widehat{\mathbb{Z}} \times \mathbb{Z} \backslash \mathbb{R} .
$$

We will be interested in $G L_{n}(\mathbb{A})$, the locally compact topological group of $n \times n$ invertible matrices with coefficients in $\mathbb{A}$. We remark that the topology on $G L_{n}(\mathbb{A})$ is the subspace topology resulting from the closed embedding

$$
\begin{aligned}
G L_{n}(\mathbb{A}) & \hookrightarrow M_{n}(\mathbb{A}) \times M_{n}(\mathbb{A}) \\
g & \mapsto\left(g, g^{-1}\right) .
\end{aligned}
$$

$G L_{n}(\mathbb{Q})$ is a discrete subgroup of $G L_{n}(\mathbb{A})$ and the quotient $G L_{n}(\mathbb{Q}) \backslash G L_{n}(\mathbb{A})$ has finite volume. If $U \subset G L_{n}(\widehat{\mathbb{Z}})$ is an open subgroup with $\operatorname{det} U=\widehat{\mathbb{Z}}^{\times}$, then

$$
G L_{n}(\mathbb{Q}) \backslash G L_{n}(\mathbb{A}) / U=\left(G L_{n}(\mathbb{Q}) \cap U\right) \backslash G L_{n}(\mathbb{R}) .
$$

Note that $G L_{n}(\mathbb{Q}) \cap U$ is a subgroup of $G L_{n}(\mathbb{Z})$ of finite index. Most of the statements we make concerning $G L_{n}(\mathbb{A})$ can be rephrased to involve only $G L_{n}(\mathbb{R})$, but at the expense of making them much more cumbersome. To achieve brevity (and because it seems more natural) we have opted to use the language of adeles. We hope that this extra abstraction will not be too confusing for the novice.

Before continuing our introduction of automorphic forms let us digress to mention class field theory, which provides a concrete example of the presentational advantages of the adelic language. It also implies essentially all the conjectures we are considering in the case of one dimensional Galois representations. Indeed this article is about the search for a non-abelian analogue of class field theory. Class field theory gives a concrete description of the abelianisation (maximal continuous 
abelian quotient) $G_{\mathbb{Q}}^{\mathrm{ab}}$ of $G_{\mathbb{Q}}$ and $W_{\mathbb{Q}_{p}}^{\mathrm{ab}}$ of $W_{\mathbb{Q}_{p}}$. First the local theory asserts that there is an isomorphism

$$
\operatorname{Art}_{p}: \mathbb{Q}_{p}^{\times} \stackrel{\sim}{\rightarrow} W_{\mathbb{Q}_{p}}^{\mathrm{ab}}
$$

with various natural properties, including the facts that $\operatorname{Art}\left(\mathbb{Z}_{p}^{\times}\right)$is the image of the inertia group $I_{\mathbb{Q}_{p}}$ in $W_{\mathbb{Q}_{p}}^{\text {ab }}$, and that the induced map

$$
\mathbb{Q}_{p}^{\times} / \mathbb{Z}_{p}^{\times} \longrightarrow W_{\mathbb{Q}_{p}}^{\mathrm{ab}} / I_{\mathbb{Q}_{p}} \subset G_{\mathbb{F}_{p}}
$$

takes $p$ to the geometric Frobenius element Frob $_{p}$. Secondly the global theory asserts that there is an isomorphism

$$
\text { Art : } \mathbb{A}^{\times} / \mathbb{Q}^{\times} \mathbb{R}_{>0}^{\times} \stackrel{\sim}{\rightarrow} G_{\mathbb{Q}}^{\mathrm{ab}}
$$

such that the restriction of Art to $\mathbb{Q}_{p}^{\times}$coincides with the composition of Art $p$ with the natural map $W_{\mathbb{Q}_{p}}^{\mathrm{ab}} \rightarrow G_{\mathbb{Q}}^{\mathrm{ab}}$. Thus Art is defined completely from a knowledge of the $\operatorname{Art}_{p}$ (and the fact that Art takes $-1 \in \mathbb{R}^{\times}$to complex conjugation) and the reciprocity theorem of global class field theory can be thought of as a determination of the kernel of $\prod_{p} \operatorname{Art}_{p}$.

We now return to our (extended) definition of automorphic forms. For each partition $n=n_{1}+n_{2}$ let $N_{n_{1}, n_{2}}$ denote the subgroup of $G L_{n}$ consisting of matrices of the form

$$
\left(\begin{array}{cc}
I_{n_{1}} & * \\
0 & I_{n_{2}}
\end{array}\right)
$$

Let $O(n) \subset G L_{n}(\mathbb{R})$ denote the orthogonal subgroup. Let $\mathfrak{z}_{n}$ denote the centre of the universal enveloping of $\mathfrak{g l}_{n}$, the complexified Lie algebra of $G L_{n}(\mathbb{R})$ (i.e. $\mathfrak{g l}_{n}=M_{n}(\mathbb{C})$ with $\left.[X, Y]=X Y-Y X\right)$. Via the Harish-Chandra isomorphism (see for example [Dix]) we may identify homomorphisms $\mathfrak{z}_{n} \rightarrow \mathbb{C}$ with multisets of $n$ complex numbers. We will write $\chi_{H}$ for the homomorphism corresponding to a multiset $H$. Thus $\mathfrak{z}_{n}$ acts on the irreducible finite dimensional $\mathfrak{g l}_{n}$-module with highest weight $\left(a_{1}, \ldots, a_{n}\right) \in \mathbb{Z}^{n}\left(a_{1} \geq \ldots \geq a_{n}\right)$ by $\chi_{\left\{a_{1}+(n-1) / 2, \ldots, a_{n}+(1-n) / 2\right\}}$.

Fix such a multiset $H$ of cardinality $n$. The space of cusp forms with infinitesimal character $H, \mathcal{A}_{H}^{\circ}\left(G L_{n}(\mathbb{Q}) \backslash G L_{n}(\mathbb{A})\right)$ is the space of smooth bounded functions

$$
f: G L_{n}(\mathbb{Q}) \backslash G L_{n}(\mathbb{A}) \longrightarrow \mathbb{C}
$$

satisfying the following conditions.

1. $\left(K\right.$-finiteness) The translates of $f$ under $G L_{n}(\widehat{\mathbb{Z}}) \times O(n)$ (where $O(n)$ denotes the orthogonal group) span a finite dimensional vector space;

2. (Infinitesimal character $H$ ) If $z \in \mathfrak{z}_{n}$ then $z f=\chi_{H}(z) f$;

3. (Cuspidality) For each partition $n=n_{1}+n_{2}$,

$$
\int_{N_{n_{1}, n_{2}}(\mathbb{Q}) \backslash N_{n_{1}, n_{2}}(\mathbb{A})} f(u g) d u=0 .
$$


Note that if $U \subset G L_{n}(\widehat{\mathbb{Z}})$ is an open subgroup with $\operatorname{det} U=\widehat{\mathbb{Z}}^{\times}$then one may think of $\mathcal{A}_{H}^{\circ}\left(G L_{n}(\mathbb{Q}) \backslash G L_{n}(\mathbb{A})\right)^{U}$ as a space of functions on $\left(G L_{n}(\mathbb{Q}) \cap U\right) \backslash G L_{n}(\mathbb{R})$.

One would like to study $\mathcal{A}_{H}^{\circ}\left(G L_{n}(\mathbb{Q}) \backslash G L_{n}(\mathbb{A})\right)$ as a representation of $G L_{n}(\mathbb{A})$, unfortunately it is not preserved by the action of $G L_{n}(\mathbb{R})$ (because the $K$-finiteness condition depends on the choice of a maximal compact subgroup $O(n) \subset G L_{n}(\mathbb{R})$ ). It does however have an action of $G L_{n}\left(\mathbb{A}^{\infty}\right) \times O(n)$ and of $\mathfrak{g l}_{n}$, which is essentially as good. More precisely it is an admissible $G L_{n}\left(\mathbb{A}^{\infty}\right) \times\left(\mathfrak{g l}_{n}, O(n)\right)$-module in the sense of [F]. In fact it is a direct sum of irreducible, admissible $G L_{n}\left(\mathbb{A}^{\infty}\right) \times\left(\mathfrak{g l}_{n}, O(n)\right)$ modules each occurring with multiplicity one. We will (slightly abusively) refer to these irreducible constituents as cuspidal automorphic representations of $G L_{n}(\mathbb{A})$ with infinitesimal character $H$.

$\mathcal{A}_{\{0\}}^{\circ}\left(\mathbb{Q}^{\times} \backslash \mathbb{A}^{\times}\right)$is just the space of locally constant functions on $\mathbb{A}^{\times} / \mathbb{Q}^{\times} \mathbb{R}_{>0}^{\times}$ and so cuspidal automorphic representations of $G L_{1}(\mathbb{A})$ with infinitesimal character $\{0\}$, are just the (finite order) complex valued characters of $\mathbb{A}^{\times} / \mathbb{Q}^{\times} \mathbb{R}_{>0}^{\times} \cong \widehat{\mathbb{Z}}^{\times}$, i.e. Dirichlet characters. $\mathcal{A}_{\{s\}}^{\circ}\left(\mathbb{Q}^{\times} \backslash \mathbb{A}^{\times}\right)$is simply obtained from $\mathcal{A}_{\{0\}}^{\circ}\left(\mathbb{Q}^{\times} \backslash \mathbb{A}^{\times}\right)$by twisting by \|\|$^{s}$, where \|\|$: \mathbb{A}^{\times} / \mathbb{Q}^{\times} \rightarrow \mathbb{R}_{>0}^{\times}$is the product of the absolute values ||$_{x}$. Thus in the case $n=1$ cuspidal automorphic representations are essentially Dirichlet characters.

The case $n=2$ is somewhat more representative. In this case we have $\mathcal{A}_{\{s, t\}}^{\circ}\left(G L_{2}(\mathbb{Q}) \backslash G L_{2}(\mathbb{A})\right)=(0)$ unless $s-t \in i \mathbb{R}, s-t \in \mathbb{Z}$ or $s-t \in(-1,1)$. It is conjectured that the third possibility can not arise unless $s=t$. Let us consider the case $s-t \in \mathbb{Z}_{>0}$ a little further. If $s-t \in \mathbb{Z}_{>0}$ then it turns out that the irreducible constituents of $\mathcal{A}_{\{s, t\}}^{\circ}\left(G L_{2}(\mathbb{Q}) \backslash G L_{2}(\mathbb{A})\right)$ are in bijection with the weight $1+s-t$ holomorphic cusp forms on the upper half plane, which are normalised newforms (see for example [Mi]). Thus in some sense cuspidal automorphic representations are are also generalisations of classical holomorphic normalised newforms.

Note that if $\psi$ is a character of $\mathbb{A}^{\times} / \mathbb{Q}^{\times} \mathbb{R}_{>0}^{\times}$and if $\pi$ is a cuspidal automorphic representation of $G L_{n}(\mathbb{A})$ with infinitesimal character $H$ then $\pi \otimes(\psi \circ$ det) is also a cuspidal automorphic representation with infinitesimal character $H$ and the contragredient (dual) $\pi^{*}$ of $\pi$ is a cuspidal automorphic representation with infinitesimal character $-H=\{-h: h \in H\}$.

One of the main questions in the theory of automorphic forms is to describe the irreducible constituents of $\mathcal{A}_{H}^{\circ}\left(G L_{n}(\mathbb{Q}) \backslash G L_{n}(\mathbb{A})\right)$. If we are to do this we first need some description of all irreducible admissible $G L_{n}\left(\mathbb{A}^{\infty}\right) \times\left(\mathfrak{g l}_{n}, O(n)\right)$-modules, and then we can try to say which occur in $\mathcal{A}_{H}^{\circ}\left(G L_{n}(\mathbb{Q}) \backslash G L_{n}(\mathbb{A})\right)$.

Just as a character $\psi: \mathbb{A}^{\times} \rightarrow \mathbb{C}^{\times}$can be factored as

$$
\psi=\psi_{\infty} \times \prod_{p} \psi_{p}
$$

where $\psi_{p}: \mathbb{Q}_{p}^{\times} \rightarrow \mathbb{C}^{\times}$(resp. $\left.\psi_{\infty}: \mathbb{R}^{\times} \rightarrow \mathbb{C}^{\times}\right)$, so an irreducible, admissible $G L_{n}\left(\mathbb{A}^{\infty}\right) \times\left(\mathfrak{g l}_{n}, O(n)\right)$-module can be factorised as a restricted tensor product (see [F])

$$
\pi \cong \bigotimes_{x}^{\prime} \pi_{x}
$$


where $\pi_{\infty}$ is an irreducible, admissible $\left(\mathfrak{g l}_{n}, O(n)\right.$ )-module (see for example $|\mathrm{Wa}|$ ), and each $\pi_{p}$ is an irreducible smooth (i.e. stabilisers of vectors are open in $G L_{n}\left(\mathbb{Q}_{p}\right)$ ) representation of $G L_{n}\left(\mathbb{Q}_{p}\right)$ with $\pi_{p}^{G L_{n}\left(\mathbb{Z}_{p}\right)} \neq(0)$ for all but finitely many $p$. To the factors $\pi_{x}$ one can associate various invariants (see $[\rrbracket]$ ).

- A central character $\psi_{x}: \mathbb{Q}_{x}^{\times} \rightarrow \mathbb{C}^{\times}$.

- L-factors $L\left(\pi_{p}, X\right) \in \mathbb{C}(X)$.

- A $\Gamma$-factor $\Gamma\left(\pi_{\infty}, s\right)$.

- Conductors $f\left(\pi_{p}\right) \in \mathbb{Z}_{\geq 0}$.

- For each non-trivial character $\Psi_{x}: \mathbb{Q}_{x} \rightarrow \mathbb{C}^{\times}$an epsilon factor $\epsilon\left(\pi_{x}, \Psi_{x}\right) \in \mathbb{C}^{\times}$.

(We also remark that $\mathfrak{z}_{n}$ acts via a character $\mathfrak{z}_{n} \rightarrow \mathbb{C}$ on any irreducible, admissible $\left(\mathfrak{g l}_{n}, O(n)\right)$-module $\pi_{\infty}$. This character is called the infinitesimal character of $\pi_{\infty}$.) Now we may attach to $\pi$

- a central character $\psi_{\pi}=\prod_{x} \psi_{x}: \mathbb{A}^{\times} \rightarrow \mathbb{C}^{\times}$;

- an $L$-function $L(\pi, s)=\prod_{p} L\left(\pi_{p}, p^{-s}\right)$ (which may or may not converge);

- an extended $L$-function $\Lambda(\pi, s)=\Gamma\left(\pi_{\infty}, s\right) L(\pi, s)$;

- a conductor $N(\pi)=\prod_{p} p^{f\left(\pi_{p}\right)}$ (which makes sense because $f\left(\pi_{p}\right)=0$ when $\left.\pi_{p}^{G L_{n}\left(\mathbb{Z}_{p}\right)} \neq(0)\right)$

- and an epsilon constant $\epsilon(\pi)=\prod_{x} \epsilon\left(\pi_{x}, \Psi_{x}\right) \in \mathbb{C}^{\times}$where $\prod_{x} \Psi_{x}: \mathbb{A} / \mathbb{Q} \rightarrow \mathbb{C}^{\times}$ is any non-trivial character.

The following theorem and conjecture describe the (expected) relationship between automorphic forms and $L$-functions with Euler product and functional equation. We suppose $n>1$. A similar theorem to theorem 3.1 is true for $n=1$, except that $L(\pi, s)$ may have one simple pole. In this case it was due to Dirichlet. Conjecture 3.2 becomes vacuous if $n=1$.

Theorem 3.1 (Godement-Jacquet, GJ]) Suppose that $\pi$ is an irreducible constituent of $\mathcal{A}_{H}^{\circ}\left(G L_{n}(\mathbb{Q}) \backslash G L_{n}(\mathbb{A})\right)$ with $n>1$. Then $L(\pi, s)$ converges to a holomorphic function in some right half complex plane $\operatorname{Re} s>\sigma$ and can be continued to a holomorphic function on the whole complex plane so that $\Lambda(\pi, s)$ is bounded in all vertical strips $\sigma_{1} \geq \operatorname{Re} s \geq \sigma_{2}$. Moreover $\Lambda(\pi, s)$ satisfies the functional equation

$$
\Lambda(\pi, s)=\epsilon(\pi) N(\pi)^{-s} \Lambda\left(\pi^{*}, 1-s\right) .
$$

Conjecture 3.2 (Cogdell-Piatetski-Shapiro, CPS1]) Suppose that $\pi$ is an irreducible, admissible $G L_{n}\left(\mathbb{A}^{\infty}\right) \times\left(\mathfrak{g l}_{n}, O(n)\right)$-module such that the central character of $\pi$ is trivial on $\mathbb{Q}^{\times}$and such that $L(\pi, s)$ converges in some half plane. Suppose also that for all characters $\psi: \mathbb{A}^{\times} / \mathbb{Q}^{\times} \mathbb{R}_{>0}^{\times} \rightarrow \mathbb{C}^{\times}$the L-function $\Lambda(\pi \otimes(\psi \circ \operatorname{det}), s)$ (which will then converge in some right half plane) can be continued to a holomorphic function on the entire complex plane, which is bounded in vertical strips and satisfies a functional equation

$\Lambda(\pi \otimes(\psi \circ \operatorname{det}), s)=\epsilon(\pi \otimes(\psi \circ \operatorname{det})) N(\pi \otimes(\psi \circ \operatorname{det}))^{-s} \Lambda\left(\pi^{*} \otimes\left(\psi^{-1} \circ \operatorname{det}\right), 1-s\right)$.

$\left(\Lambda\left(\pi^{*} \otimes\left(\psi^{-1} \circ \operatorname{det}\right), s\right)\right.$ also automatically converges in some right half plane.) Then there is a partition $n=n_{1}+\ldots+n_{r}$ and cuspidal automorphic representations $\pi_{i}$ 
of $G L_{n_{i}}(\mathbb{A})$ such that

$$
\Lambda(\pi, s)=\prod_{i=1}^{r} \Lambda\left(\pi_{i}, s\right)
$$

This conjecture is known to be true for $n=2(\sqrt{\mathrm{We}}$, , JU $)$ and $n=3$ ( For $n>3$ a weaker form of this conjecture involving twisting by higher dimensional automorphic representations is known to hold (see [CPS1], [CPS2]). These results are called 'converse theorems'.

The reason for us introducing automorphic forms is because of a putative connection to Galois representations, which we will now discuss. But first let us discuss the local situation. It has recently been established ( $[\mathrm{HT}],[\mathrm{He}]$, Ha $)$ that there is a natural bijection, $\operatorname{rec}_{p}$, from irreducible smooth representations of $G L_{n}\left(\mathbb{Q}_{p}\right)$ to $n$-dimensional Frobenius semi-simple WD-representations of $W_{\mathbb{Q}_{p}}$ over $\mathbb{C}$. The key point here is that the bijection should be natural. We will not describe here exactly what this means (instead we refer the reader to the introduction to [HT]). It does satisfy the following.

- $\psi_{\pi} \circ \operatorname{Art}_{p}^{-1}=\operatorname{det} \operatorname{rec}_{p}(\pi)$, where $\psi_{\pi}$ is the central character of $\pi$.

- $L\left(\operatorname{rec}_{p}(\pi), X\right)=L(\pi, X)$.

- $f\left(\operatorname{rec}_{p}(\pi)\right)=f(\pi)$.

- $\epsilon\left(\operatorname{rec}_{p}(\pi), \Psi_{p}\right)=\epsilon\left(\pi, \Psi_{p}\right)$ for any non-trivial character $\Psi_{p}: \mathbb{Q}_{p} \rightarrow \mathbb{C}^{\times}$.

The existence of $\operatorname{rec}_{p}$ can be seen as a non-abelian generalisation of local class field theory, as in the case $n=1$ we have $\operatorname{rec}_{p}(\pi)=\pi \circ \operatorname{Art}_{p}^{-1}$.

Now suppose that $\iota: \overline{\mathbb{Q}}_{l} \rightarrow \mathbb{C}$ and that $R$ is a de Rham $l$-adic representation of $G_{\mathbb{Q}}$ which is unramified at all but finitely many primes. Using the local reciprocity map $\operatorname{rec}_{p}$, we can associate to $R$ an irreducible, admissible $G L_{n}\left(\mathbb{A}^{\infty}\right) \times\left(\mathfrak{g l}_{n}, O(n)\right)$ module

$$
\pi(\iota R)=\pi_{\infty}(R) \otimes \prod_{p} \operatorname{rec}_{p}^{-1}\left(\iota \mathrm{WD}_{p}(R)\right),
$$

where $\pi_{\infty}(R)$ is a tempered irreducible, admissible $\left(\mathfrak{g l}_{n}, O(n)\right)$-module with infinitesimal character $\operatorname{HT}\left(\left.R\right|_{G_{\mathbb{Q}_{l}}}\right)$ and with $\Gamma\left(\pi_{\infty}(R), s\right)=\Gamma(R, s)$. The definition of $\pi_{\infty}(R)$ depends only on the numbers $m_{i}^{R}$ and $m_{w / 2, \pm}^{R}$. Then we have the following conjectures.

Conjecture 3.3 Suppose that $H$ is a multiset of $n$ integers and that $\pi$ is an irreducible constituent of $\mathcal{A}_{H}^{\circ}\left(G L_{n}(\mathbb{Q}) \backslash G L_{n}(\mathbb{A})\right)$. Identify $\overline{\mathbb{Q}} \subset \mathbb{C}$. Then each $\operatorname{rec}_{p}\left(\pi_{p}\right)$ can be defined over $\overline{\mathbb{Q}}$ and there is an irreducible geometric strongly compatible system of $l$-adic representations $\mathcal{R}$ such that $\mathrm{HT}(\mathcal{R})=H$ and $\mathrm{WD}_{p}(\mathcal{R})^{\mathrm{ss}}=\operatorname{rec}_{p}\left(\pi_{p}\right)$ for all primes $p$.

\section{Conjecture 3.4 Suppose that}

$$
R: G_{\mathbb{Q}} \longrightarrow G L(V)
$$

is an irreducible $l$-adic representation which is unramified at all but finitely many primes and for which $\left.R\right|_{G_{\mathbb{Q}_{l}}}$ is de Rham. Let $\iota: \overline{\mathbb{Q}}_{l} \rightarrow \mathbb{C}$. Then $\pi(\iota R)$ is a cuspidal automorphic representation of $G L_{n}(\mathbb{A})$. 
These conjectures are essentially due to Langlands Lan1], except we have used a precise formulation which follows Clozel Cl1 and we have incorporated conjecture 1.1 into conjecture 3.4.

Conjecture 3.4 is probably the more mysterious of the two, as only the case $n=1$ and fragmentary cases where $n=2$ are known. This will be discussed further in the next section. Note the similarity to the main theorem of global class field theory that $\prod_{p} \operatorname{Art}_{p}: \mathbb{A}^{\times} \rightarrow G_{\mathbb{Q}}^{\text {ab }}$ has kernel $\mathbb{Q}^{\times}$.

The following theorem provides significant evidence for conjecture 3.3.

Theorem 3.5 ([Kot], [C12], [HT]) Suppose that $H$ is multiset of $n$ distinct integers and that $\pi$ is an irreducible constituent of $\mathcal{A}_{H}^{\circ}\left(G L_{n}(\mathbb{Q}) \backslash G L_{n}(\mathbb{A})\right)$. Let $\iota: \overline{\mathbb{Q}}_{l} \hookrightarrow \mathbb{C}$. Suppose moreover that $\pi^{*} \cong \pi \otimes(\psi \circ$ det $)$ for some character $\psi: \mathbb{A}^{\times} / \mathbb{Q}^{\times} \rightarrow \mathbb{C}^{\times}$, and that either $n \leq 2$ or for some prime $p$ the representation $\pi_{p}$ is square integrable (i.e. $\operatorname{rec}_{p}\left(\pi_{p}\right)$ is indecomposable). Then there is a continuous representation

$$
R_{l, \iota}: G_{\mathbb{Q}} \longrightarrow G L_{n}\left(\overline{\mathbb{Q}}_{l}\right)
$$

with the following properties.

1. $R_{l, \iota}$ is geometric and pure of weight $2 / n \sum_{h \in H} h$.

2. $\left.R_{l, \iota}\right|_{G_{\mathbb{Q}_{l}}}$ is de Rham and $\mathrm{HT}\left(\left.R_{l, l}\right|_{G_{\mathbb{Q}_{l}}}\right)=H$.

3. For any prime $p \neq l$ there is a representation $r_{p}: W_{\mathbb{Q}_{p}} \rightarrow G L_{n}\left(\overline{\mathbb{Q}}_{l}\right)$ such that $\mathrm{WD}_{p}\left(R_{l, \iota}\right)^{\mathrm{ss}}=\left(r_{p}, N_{p}\right)$ and $\operatorname{rec}_{p}\left(\pi_{p}\right)=\left(\iota r_{p}, N_{p}^{\prime}\right)$.

This was established by finding the desired $l$-adic representations in the cohomology of certain unitary group Shimura varieties. It seems not unreasonable to hope that similar techniques might allow one to improve many of the technical defects in the theorem. However Clozel has stressed that in the cases where $H$ does not have distinct elements or where $\pi^{*} \not \approx \pi \otimes(\psi \circ$ det $)$, there seems to be no prospect of finding the desired $l$-adic representations in the cohomology of Shimura varieties. It seems we need a new technique.

\section{What do we know?}

Let us first summarise in a slightly less precise way the various conjectures we have made, in order to bring together the discussion so far. Fix an embedding $\overline{\mathbb{Q}} \hookrightarrow \mathbb{C}$ and let $H$ be a multiset of integers of cardinality $n>1$. Then the following sets should be in natural bijection. One way to make precise the meaning of 'natural' is to require that two objects $M$ and $M^{\prime}$ should correspond if the local L-factors $L_{p}(M, X)$ and $L_{p}\left(M^{\prime}, X\right)$ are equal for all but finitely many $p$. Note that in each case the factors $L_{p}(M, X)$ for all but finitely many $p$, completely determine $M$.

(AF) Irreducible constituents $\pi$ of $\mathcal{A}_{H}^{\circ}\left(G L_{n}(\mathbb{Q}) \backslash G L_{n}(\mathbb{A})\right)$.

(LF) Near equivalence classes of irreducible, admissible $G L_{n}\left(\mathbb{A}^{\infty}\right) \times\left(\mathfrak{g l}_{n}, O(n)\right)$ modules $\pi$ with the following properties. (We call two $G L_{n}\left(\mathbb{A}^{\infty}\right) \times\left(\mathfrak{g l} l_{n}, O(n)\right)$ modules, $\pi$ and $\pi^{\prime}$ nearly equivalent if $\pi_{p} \cong \pi_{p}^{\prime}$ for all but finitely many $p$.) 
(a) $\pi_{\infty}$ has infinitesimal character $H$.

(b) The central character $\psi_{\pi}$ is trivial on $\mathbb{Q}^{\times} \subset \mathbb{A}^{\times}$.

(c) For all characters $\psi: \mathbb{A}^{\times} / \mathbb{Q}^{\times} \mathbb{R}_{>0}^{\times}$the $L$-function $\Lambda(\pi \otimes(\psi \circ \operatorname{det}), s)$ converges in some right half plane, has holomorphic continuation to the entire complex plane so that it is bounded in vertical strips and satisfies the functional equation

$$
\Lambda(\pi \otimes \psi, s)=\epsilon(\pi \otimes \psi) N(\pi \otimes \psi)^{-s} \Lambda\left(\pi^{*} \otimes \psi^{-1}, 1-s\right) .
$$

(d) (See JS for an explanation of this condition.) There is a finite set of primes $S$ containing all primes $p$ for which $\operatorname{rec}_{p}\left(\pi_{p}\right)$ is ramified, such that, writing $L\left(\pi_{p}, X\right)=\prod_{i=1}^{n}\left(1-\alpha_{p, i} X\right)^{-1}$ for $p \notin S$,

$$
\sum_{p \notin S, i, j} \sum_{m=1}^{\infty} \alpha_{p, i}^{m} \alpha_{p, j}^{-m} / m p^{m s}+\log (s-1)
$$

is bounded as $s \rightarrow 1$ from the right.

In this case $L_{p}(\pi, X)=L\left(\pi_{p}, X\right)$.

(IR) $\left(\right.$ Fix $\iota: \overline{\mathbb{Q}}_{l} \rightarrow \mathbb{C}$.) Irreducible $l$-adic representations

$$
R: G_{\mathbb{Q}} \longrightarrow G L_{n}\left(\overline{\mathbb{Q}}_{l}\right)
$$

which are unramified at all but finitely many primes and for which $\left.R\right|_{G_{\mathbb{Q}_{l}}}$ is de Rham with $\operatorname{HT}\left(\left.R\right|_{G_{\mathbb{Q}_{l}}}\right)=H$. In this case $L_{p}(R, X)=$ $\iota L\left(\mathrm{WD}_{p}(R), X\right)$.

(WCS) Irreducible weakly compatible systems of $l$-adic representations $\mathcal{R}$ with

$\mathrm{HT}(\mathcal{R})=H$. In this case $L_{p}(\mathcal{R}, X)=L_{p}\left(\mathrm{WD}_{p}(\mathcal{R}), X\right)$.

(GCS) Irreducible geometric strongly compatible systems of $l$-adic representations $\mathcal{R}$ with $\operatorname{HT}(\mathcal{R})=H$. In this case $L_{p}(\mathcal{R}, X)=L_{p}\left(\mathrm{WD}_{p}(\mathcal{R}), X\right)$.

For $n=1$ we must drop the item $(L F)$, because it would need to be modified to allow $L(\pi \otimes(\psi \circ$ det $), s)$ to have a simple pole, while, in any case condition (LF) (b) would make the implication $(L F) \Longrightarrow(A F)$ trivial. This being said, in the case $n=1$ all the other four sets are known to be in natural bijection (see [Se]). This basically follows because global class field theory provides an isomorphism

$$
\text { Art }: \mathbb{A}^{\times} / \mathbb{Q}^{\times} \mathbb{R}_{>0}^{\times} \stackrel{\sim}{\longrightarrow} G_{\mathbb{Q}}^{\mathrm{ab}} .
$$

I would again like to stress how different are these various sorts of objects and how surprising it is to me that there is any relation between them. Items (AF) and (LF) both concern representations of adele groups, but arising in rather different settings: either from the theory of discrete subgroups of Lie groups or from the 
theory of $L$-functions with functional equation. Items (IR) and (WCS) arise from Galois theory and item (GCS) arises from geometry.

So what do we know about the various relationships for $n>1$ ?

Not much. Trivially one has $(G C S) \Longrightarrow(W C S) \Longrightarrow(l R)$. The passage $(A F) \Longrightarrow(L F)$ is OK by theorem 3.1. As discussed in section 3 we have significant partial results in the directions $(L F) \Longrightarrow(A F)$ and $(A F) \Longrightarrow(G C S)$, but both seem to need new ideas. (Though I should stress that I am not really competent to discuss converse theorems.)

One way to establish the equivalence of all five items would be to complete the passages $(L F) \Longrightarrow(A F)$ and $(A F) \Longrightarrow(G C S)$ and to establish the passage $(l R) \Longrightarrow(A F)$. It is these implications which have received most study, though it should be pointed out that in the function field case the equivalence of the analogous objects was established by looking at the implications

$$
(l R) \Longrightarrow(L F) \Longrightarrow(A F) \Longrightarrow(G C S) .
$$

(See [Lat]. It is the use of techniques from Grothendieck's $l$-adic cohomology to prove the first of these implications which is most special to function fields.) However it is striking that in the case of number fields all known implications from items (lR), (WCS) or (GCS) to (LF) go via (AF).

For the rest of this article we will concentrate on what still seems to be the least understood problem: the passage from (IR) or (WCS) to (AF) or (LF). Although the results we have are rather limited one should not underestimate their power. Perhaps the most striking illustration of this is that the lifting theorems discussed in section 4.4 (combined with earlier work using base change and converse theorems) allowed Wiles Wi] to finally prove Fermat's last theorem.

The discussion in the rest of this paper will of necessity be somewhat more technical. In particular we will need to discuss automorphic forms, $l$-adic representations and so on over general number fields (i.e. fields finite over $\mathbb{Q}$ ). We will leave it to the reader's imagination exactly how such a generalisation is made. In this connection we should remark that if $L / K$ is a finite extension of number fields and if $R$ is a semi-simple de Rham $l$-adic representation of $G_{L}$ which is unramified at all but finitely many primes, then (see [A])

$$
L(R, s)=L\left(\operatorname{Ind}_{G_{L}}^{G_{K}} R, s\right)
$$

(formally if the $L$-functions don't converge). In fact this is true Euler factor by Euler factor and similar results hold for conductors and $\epsilon$-factors (see Tat). This observation can be extremely useful.

\subsection{Cyclic base change}

Suppose that $G$ is a group, $H$ a normal subgroup such that $G / H$ is cyclic with generator of $\sigma$. It is an easy exercise that an irreducible representation $r$ of $H$ extends to a representation of $G$ if and only if $r^{\sigma} \cong r$ as representations of $H$. If one believes conjectures 3.3 and 3.4, one might expect that if $L / K$ is a cyclic Galois extension of number fields of prime order, if $\sigma$ generates $\operatorname{Gal}(L / K)$ and if $\pi$ is a 
cuspidal automorphic representation of $G L_{n}\left(\mathbb{A}_{L}\right)$ with $\pi \circ \sigma \cong \pi$, then there should be a cuspidal automorphic representation $\Pi$ of $G L_{n}\left(\mathbb{A}_{K}\right)$, such that for all places $w$ of $L$ we have $\operatorname{rec}_{w}\left(\pi_{w}\right)=\left.\operatorname{rec}_{\left.w\right|_{K}}\left(\Pi_{\left.w\right|_{K}}\right)\right|_{W_{L_{w}}}$. This is indeed the case. For $n=1$ we have $\pi=\Pi \circ \mathbf{N}_{L / K}$, Langlands Lan2 proved it for $n=2$ using the trace formula and Arthur and Clozel $\mathrm{AC}$ generalised his method to all $n$.

One drawback of this result is that if $v$ is a place of $K$ inert in $L$ then there is no complete recipe for $\Pi_{v}$ in terms of $\pi$. This can be surprisingly serious. It can however be alleviated, if we know how to associate irreducible $l$-adic representations to both $\Pi$ and $\pi$. Langlands used this to show that many two dimensional Artin representations (i.e. $l$-adic representations with finite image) were automorphic (i.e. associated to a cuspidal automorphic representation). In fact using additional results from the theory of $L$-functions, particularly the converse theorem for $G L_{3}$ (see section 4.3), he and Tunnell ([Tu]) were able to establish the automorphy of all continuous two dimensional Artin representations with soluble image.

\subsection{Brauer's theorem}

The result I want to discuss is a result of Brauer $[\mathrm{Br}]$ about finite groups.

Theorem 4.1 (Brauer) Suppose that $r$ is a representation of a finite group $G$. Then there are soluble subgroups $H_{i}<G$, one dimensional representations $\psi_{i}$ of $H_{i}$ and integers $n_{i}$ such that as virtual representations of $G$ we have

$$
r=\sum_{i} n_{i} \operatorname{Ind}_{H_{i}}^{G} \psi_{i}
$$

As Artin A] had realised this theorem has the following immediate consequence. (Indeed Brauer proved his theorem in response to Artin's work.)

Corollary 4.2 Let $\iota: \overline{\mathbb{Q}}_{l} \rightarrow \mathbb{C}$. Suppose that

$$
R: G_{\mathbb{Q}} \longrightarrow G L_{n}\left(\overline{\mathbb{Q}}_{l}\right)
$$

is an l-adic representation with finite image. Then the $L$-function $L(\iota R, s)$ has meromorphic continuation to the entire complex plane and satisfies the expected functional equation.

Artin's argument runs as follows. Let $G$ denote the image of $R$ and write

$$
R=\sum_{i} n_{i} \operatorname{Ind}_{H_{i}}^{G} \psi_{i}
$$

as in Brauer's theorem. Let $L / \mathbb{Q}$ be the Galois extension with group $G$ cut out by $R$ and let $K_{i}=L^{H_{i}}$. Then one has equalities

$$
\begin{aligned}
L(\iota R, s) & =\prod_{i} L\left(\iota \operatorname{Ind}_{G_{K_{i}}}^{G_{Q}} \psi_{i}, s\right)^{n_{i}} \\
& =\prod_{i} L\left(\iota \psi_{i}, s\right)^{n_{i}} .
\end{aligned}
$$


By class field theory for the fields $K_{i}$, the character $\psi_{i}$ is automorphic on $G L_{1}\left(\mathbb{A}_{K_{i}}\right)$ and so $L\left(\iota \psi_{i}, s\right)$ has holomorphic continuation to the entire complex plane (except possibly for one simple pole if $\psi_{i}=1$ ) and satisfies a functional equation. It follows that $L(\iota R, s)$ has meromorphic continuation to the entire complex plane and satisfies a functional equation. The problem with this method is that some of the integers $n_{i}$ will usually be negative so that one can only conclude the meromorphy of $L(\iota R, s)$, not its holomorphy.

\subsection{Converse theorems}

As Cogdell and Piatetski-Shapiro point out, conjecture 3.2 would have very important implications for Galois representations. For instance the cases $n=2$ and 3 played a key role in the proof of the automorphy of two dimensional Artin representations (see 4.1). Conjecture 3.2 combined with Brauer's theorem and a result of Jacquet and Shalika [JS] in fact implies that many (all? - certainly those with soluble or perfect image) Artin representations are automorphic. A similar argument shows that in many other cases, in order to check the automorphy of an $l$-adic representation of $G_{\mathbb{Q}}$, it suffices to do so after a finite base change. For instance one has the following result.

Assume conjecture 3.9. Let $\iota: \overline{\mathbb{Q}}_{l} \hookrightarrow \mathbb{C}$ and let $K / \mathbb{Q}$ be a finite, totally real Galois extension. Suppose that $\Pi$ is a cuspidal automorphic representation of $G L_{n}\left(\mathbb{A}_{K}\right)$ with infinitesimal character corresponding to a multiset $H$ consisting of $n$ distinct integers. If $n>2$ also suppose that $\Pi_{v}$ is square integrable (i.e. $\operatorname{rec}_{v}\left(\Pi_{v}\right)$ is indecomposable) for some finite place $v$ of $K$. Let

$$
R: G_{\mathbb{Q}} \longrightarrow G L_{n}\left(\overline{\mathbb{Q}}_{l}\right)
$$

be an $l$-adic representation such that $R \sim R^{*} \otimes \psi$ for some character $\psi$ of $G_{\mathbb{Q}}$, and such that $\left.R\right|_{G_{K}}$ is irreducible. Suppose finally that $\left.R\right|_{G_{K}}$ and $\Pi$ are associated, in the sense that, for all but finitely many places $v$ of $K$, we have

$$
\iota L\left(\mathrm{WD}_{v}\left(\left.R\right|_{G_{K}}\right), X\right)=L\left(\Pi_{v}, X\right) .
$$

Then there is a regular algebraic cuspidal automorphic representation $\pi$ of $G L_{n}(\mathbb{A})$ associated to $R$ in the same sense.

\subsection{Lifting theorems}

To describe this sort of theorem we first remark that if $R: G_{\mathbb{Q}} \rightarrow G L_{n}\left(\overline{\mathbb{Q}}_{l}\right)$ is continuous then after conjugating $R$ by some element of $G L_{n}\left(\overline{\mathbb{Q}}_{l}\right)$ we may assume that the image of $R$ is contained in $G L_{n}\left(\mathcal{O}_{\overline{\mathbb{Q}}_{l}}\right)$ and so reducing we obtain a continuous representation

$$
\bar{R}: G_{\mathbb{Q}} \longrightarrow G L_{n}\left(\overline{\mathbb{F}}_{l}\right) .
$$

The lifting theorems I have in mind are results of the general form if $R$ and $R^{\prime}$ are $l$-adic representations of $G_{\mathbb{Q}}$ with $R^{\prime}$ automorphic and if $\bar{R}=\bar{R}^{\prime}$ then $R$ is also automorphic. Very roughly speaking the technique (pioneered by Wiles [Wi and 
completed by the author and Wiles [TW]) is to show that $R \bmod l^{r}$ arises from automorphic forms for all $r$ by induction on $r$. As $\operatorname{ker}\left(G L_{n}\left(\mathbb{Z} / l^{r} \mathbb{Z}\right) \rightarrow G L_{n}\left(\mathbb{Z} / l^{r-1} \mathbb{Z}\right)\right)$ is an abelian group one is led to questions of class field theory and Galois cohomology.

I should stress that such theorems are presently available only in very limited situations. I do not have the space to describe the exact limitations, which are rather technical, but the sort of restrictions that are common are as follows.

1. If $R: G_{\mathbb{Q}} \rightarrow G L(V)$ then there should be a character $\mu: G_{\mathbb{Q}} \rightarrow G L_{n}\left(\overline{\mathbb{Q}}_{l}\right)$ and a non-degenerate bilinear form ( , ) on $V$ such that

- $\left(R(\sigma) v_{1}, R(\sigma) v_{2}\right)=\mu(\sigma)\left(v_{1}, v_{2}\right)$ and

- $\left(v_{2}, v_{1}\right)=\mu(c)\left(v_{1}, v_{2}\right)$.

(This seems to be essential for the method of [TW].)

2. $R$ should be de Rham with distinct Hodge-Tate numbers. (This again seems essential to the method of [TW], but see [BT].)

3. Either $R$ and $R^{\prime}$ should be ordinary (i.e. their restrictions to $G_{\mathbb{Q}_{l}}$ should be contained in a Borel subgroup); or $R$ and $R^{\prime}$ should be crytsalline (not just de Rham) at $l$ with the same Hodge-Tate numbers and $l$ should be large compared with the differences of elements of $\operatorname{HT}(R)$. (The problems here are connected with the need for an integral Fontaine theory, but they are not simply technical problems. There are some complicated results pushing back this restriction in isolated cases, see [CDT], [BCDT], [Sa], but so far our understanding is very limited.)

4. The image of $\bar{R}$ should not be too small (e.g. should be irreducible when restricted to $\mathbb{Q}\left(e^{2 \pi i / l}\right)$ ), though in the case $n=2$ there is beautiful work of Skinner and Wiles (SW1 and [SW3] dispensing with this criterion, which this author has unfortunately not fully understood.

In addition, all the published work is for the case $n=2$. However there is ongoing work of a number of people attempting to dispense with this assumption. Using a very important insight of Diamond [Dia], the author, together with L.Clozel and M.Harris, has generalised to all $n$ the so called minimal case (originally treated in [TW] where $R$ is no more ramified than $\bar{R}$. One would hope to be able to deduce the non-minimal case from this, as Wiles did in Wi] for $n=2$. In this regard one should note the work of Skinner and Wiles SW2 and the work of Mann [Ma]. However there seems to be one missing ingredient, the analogue of the ubiquitous Ihara lemma, see lemma 3.2 of [Ih] (and also theorem 4.1 of [R]). As this seems to be an important question, but one which lies in the theory of discrete subgroups of Lie groups, let us take the trouble to formulate it, in the hope that an expert may be able to prove it. It should be remarked that there are a number of possible formulations, which are not completely equivalent and any of which would seem to suffice. We choose to present one which has the virtue of being relatively simple to state.

Conjecture 4.3 Suppose that $G / \mathbb{Q}$ is a unitary group which becomes an inner form of $G L_{n}$ over an imaginary quadratic field $E$. Suppose that $G(\mathbb{R})$ is compact. Let $l$ be a prime which one may assume is large compared to $n$. Let $p_{1}$ and $p_{2}$ be distinct primes different from l with $G\left(\mathbb{Q}_{p_{1}}\right) \cong G L_{n}\left(\mathbb{Q}_{p_{1}}\right)$ and $G\left(\mathbb{Q}_{p_{2}}\right) \cong G L_{n}\left(\mathbb{Q}_{p_{2}}\right)$. 
Let $U$ be an open compact subgroup of $G\left(\mathbb{A}^{p_{1}, p_{2}}\right)$ and consider the representation of $G L_{n}\left(\mathbb{Q}_{p_{1}}\right) \times G L_{n}\left(\mathbb{Q}_{p_{2}}\right)$ on the space $C^{\infty}\left(G(\mathbb{Q}) \backslash G(\mathbb{A}) / U, \overline{\mathbb{F}}_{l}\right)$ of locally constant $\overline{\mathbb{F}}_{l}$-valued functions on

$$
G(\mathbb{Q}) \backslash G(\mathbb{A}) / U=(G(\mathbb{Q}) \cap U) \backslash\left(G L_{n}\left(\mathbb{Q}_{p_{1}}\right) \times G L_{n}\left(\mathbb{Q}_{p_{2}}\right) .\right.
$$

(Note that $G(\mathbb{Q}) \cap U$ is a discrete cocompact subgroup of $G L_{n}\left(\mathbb{Q}_{p_{1}}\right) \times G L_{n}\left(\mathbb{Q}_{p_{2}}\right)$.) Suppose that $\pi_{1} \otimes \pi_{2}$ is an irreducible sub-representation of $C^{\infty}\left(G(\mathbb{Q}) \backslash G(\mathbb{A}) / U, \overline{\mathbb{F}}_{l}\right)$ with $\pi_{1}$ generic. Then $\pi_{2}$ is also generic.

The most serious problem with applying such lifting theorems to prove an $l$ adic representation $R$ is automorphic is the need to find some way to show that $\bar{R}$ is automorphic. The main success of lifting theorems to date, has been to show that if $E$ is an elliptic curve over the rationals then $H^{1}\left(E(\mathbb{E}), \overline{\mathbb{Q}}_{l}\right)$ is automorphic, so that $E$ is a factor of the Jacobian of a modular curve and the $L$-function $L(E, s)$ is an entire function satisfying the expected functional equation ([W], [TW], [BCDT]). This was possible because $G L_{2}\left(\mathbb{Z}_{3}\right)$ happens to be a pro-soluble group and there is a homomorphism $G L_{2}\left(\mathbb{F}_{3}\right) \longrightarrow G L_{2}\left(\mathbb{Z}_{3}\right)$ splitting the reduction map. The Artin representation

$$
G_{\mathbb{Q}} \longrightarrow G L\left(H^{1}\left(E(\mathbb{C}), \mathbb{F}_{3}\right)\right) \longrightarrow G L_{2}\left(\mathbb{Z}_{3}\right)
$$

is automorphic by the Langlands-Tunnell theorem alluded to in section 4.1.

\subsection{Other techniques?}

I would like to discuss one other technique which has been some help if $n=2$ and may be helpful more generally. We will restrict our attention here to the case $n=2$ and $\operatorname{det} R(c)=-1$. We have said that the principal problem with lifting theorems for proving an $l$-adic representation $R: G_{\mathbb{Q}} \rightarrow G L_{2}\left(\overline{\mathbb{Q}}_{l}\right)$ is automorphic is that one needs to know that $\bar{R}$ is automorphic. This seems to be a very hard problem. Nonetheless one can often show that $\bar{R}$ becomes automorphic over some Galois totally real field $K / \mathbb{Q}$. (Because $K$ is totally real, if $\bar{R}\left(G_{\mathbb{Q}}\right) \supset S L_{2}\left(\mathbb{F}_{l}\right)$ and $l>3$ then $\bar{R}\left(G_{K}\right) \supset S L_{2}\left(\mathbb{F}_{l}\right)$. So this 'potential automorphy' is far from vacuous). The way one does this is to look for an abelian variety $A / K$ with multiplication by a number field $F$ with $[F: \mathbb{Q}]=\operatorname{dim} A$, and such that $\bar{R}$ is realised on $H^{1}\left(A(\mathbb{C}), \mathbb{F}_{l}\right)[\lambda]$ for some prime $\lambda \mid l$, while for some prime $\lambda^{\prime} \mid l^{\prime} \neq l$ the image of $G_{K}$ on $H^{1}\left(A(\mathbb{C}), \mathbb{F}_{l^{\prime}}\right)\left[\lambda^{\prime}\right]$ is soluble. One then argues that $H^{1}\left(A(\mathbb{C}), \mathbb{F}_{l^{\prime}}\right)\left[\lambda^{\prime}\right]$ is automorphic, hence by a lifting theorem $H^{1}\left(A(\mathbb{C}), \mathbb{Q}_{l^{\prime}}\right) \otimes_{F_{l^{\prime}}} F_{\lambda^{\prime}}$ is automorphic, so that (tautologically) $H^{1}\left(A(\mathbb{C}), \mathbb{F}_{l}\right)[\lambda]$ is also automorphic, and hence, by another lifting theorem, $\left.R\right|_{G_{K}}$ is automorphic. One needs $K$ to be totally real, as over general number fields there seems to be no hope of proving lifting theorems, or even of attaching $l$-adic representations to automorphic forms. In practice, because of various limitations in the lifting theorems one uses, one needs to impose some conditions on the behaviour of a few primes, like $l$, in $K$ and some other conditions on $A$. The problem of finding a suitable $A$ over a totally real field $K$, comes down to finding a $K$-point on a twisted Hilbert modular variety. This is possible because we are free to choose $K$, the only restriction being that $K$ is totally real and certain small 
primes (almost) split completely in $K$. To do this, one has the following relatively easy result.

Proposition $4.4(\sqrt{\mathrm{MB}}, \mid \mathrm{P}])$ Suppose that $X / \mathbb{Q}$ is a smooth geometrically irreducible variety. Let $S$ be a finite set of places of $\mathbb{Q}$ and suppose that $X$ has a point over the completion of $\mathbb{Q}$ at each place in $S$. Let $\mathbb{Q}_{S}$ be the maximal extension of $\mathbb{Q}$ in which all places in $S$ split completely (e.g. $\mathbb{Q}_{\{\infty\}}$ is the maximal totally real field). Then $X$ has a $\mathbb{Q}_{S}$-point.

In this regard it would have extremely important consequences if, in the previous proposition, one could replace $\mathbb{Q}_{S}$ by $\mathbb{Q}_{S}^{\text {sol }}$, the maximal soluble extension of $\mathbb{Q}$ in which all places in $S$ split completely. I do not know if it is reasonable to expect this.

Using this method one can, for instance, prove the following result.

Theorem 4.5 ([Tay]) Suppose that $\mathcal{R}$ is an irreducible weakly compatible system of two dimensional l-adic representations with $\operatorname{HT}(\mathcal{R})=\left\{n_{1}, n_{2}\right\}$ where $n_{1} \neq n_{2}$. Suppose also that $\operatorname{det} R_{l, \iota}(c)=-1$ for one (and hence for all) pairs $(l, \iota)$. Then there is a Galois totally real field $K / \mathbb{Q}$ and a cuspidal automorphic representation $\pi$ of $G L_{2}\left(\mathbb{A}_{K}\right)$ such that

- for all $v \mid \infty, \pi_{v}$ has infinitesimal character $H$, and

- for all $(l, \iota)$ and for all finite places $v$ Xl of $K$ we have

$$
\operatorname{rec}_{v}\left(\pi_{v}\right)=\mathrm{WD}_{v}\left(\left.R_{l, \iota}\right|_{G_{K}}\right)^{\mathrm{ss}} .
$$

In particular $\mathcal{R}$ is pure of weight $\left(n_{1}+n_{2}\right) / 2$. Moreover $\mathcal{R}$ is strongly compatible and $L(\iota \mathcal{R}, s)$ has meromorphic continuation to the entire complex plane and satisfies the expected functional equation.

The last sentence of this theorem results from the first part and Brauer's theorem. We remark that conjecture 3.2 would imply that this theorem could be improved to assert the automorphy of $\mathcal{R}$ over $\mathbb{Q}$.

\section{References}

[A] E.Artin, Zur Theorie der L-Reihen mit allgemeinen Gruppencharakteren, Abh. Math. Sem. Univ. Hamburg 8 (1930), 292-306.

[AC] J.Arthur and L.Clozel, Simple algebras, base change and the advanced theory of the trace formula, Annals of Math. Studies 120, PUP 1989.

[Berg] L.Berger, Représentations p-adiques et équations différentielles, Invent. math. 148 (2002), 219-284.

[Bert] P.Berthelot, Altérations de variétés algébriques (d'après A.J.de Jong), Astérisque 241 (1997), 273-311.

[BCDT] C.Breuil, B.Conrad, F.Diamond and R.Taylor, On the modularity of elliptic curves over $\mathbb{Q}$, J.A.M.S. 14 (2001), 843-939.

[Br] R.Brauer, On Artin's L-series with general group characters, Ann. Math. (2) 48 (1947), 502-514. 
[BSD] B.Birch and P.Swinnerton-Dyer, Notes on elliptic curves II, J. Reine Angew. Math. 218 (1965), 79-108.

[BT] K.Buzzard and R.Taylor, Companion forms and weight one forms, Annals of math 149 (1999), 905-919.

[CDT] B.Conrad, F.Diamond and R.Taylor, Modularity of certain potentially Barsotti-Tate Galois representations, JAMS 12 (1999), 521-567.

[Cl1] L.Clozel, Motifs et formes automorphes: applications du principe de fonctorialité, in "Automorphic forms, Shimura varieties and $L$-functions I", Academic Press 1990.

[Cl2] L.Clozel, Représentations Galoisiennes associées aux representations automorphes autoduales de GL(n), Pub. Math. IHES 73 (1991), 97-145.

[CPS1] J.Cogdell and I.Piatetski-Shapiro, Converse theorems for $G L_{n}$, Publ. Math. IHES 79 (1994), 157-214.

[CPS2] J.Cogdell and I.Piatetski-Shapiro, Converse theorems for $G L_{n} I I$, J. reine angew. Math. 507 (1999), 165-188.

[De] P.Deligne, La conjecture de Weil I, Publ. Math. IHES 43 (1974), 273-307.

[Dia] F.Diamond, The Taylor-Wiles construction and multiplicity one, Invent. Math. 128 (1997), 379-391.

[Dix] J.Dixmier, Algèbres enveloppantes, Gauthier Villars 1974.

[Fa] G.Faltings, Endlichkeitssätze für abelsche Varietäten über Zahlkörpern, Invent. math. 73 (1983), 349-366.

[Fl] D.Flath, Decomposition of representations into tensor products, in "Automorphic forms, representations and $L$-functions I" AMS 1979.

[Fo1] J.-M.Fontaine, talk at "Mathematische Arbeitstagung 1988", Maz-PlanckInstitut für Mathematik preprint no. 30 of 1988.

[Fo2] J.-M.Fontaine, Le corps des périodes p-adiques, Astérisque 223 (1994), $59-111$.

[Fo3] J.-M.Fontaine, Représentations p-adiques semi-stables, Astérisque 223 (1994), 113-184.

[Fo4] J.-M.Fontaine, Représentations l-adiques potentiellement semi-stables, Astérisque 223 (1994), 321-347.

[FM] J.-M.Fontaine and B.Mazur, Geometric Galois representations, in Elliptic curves, modular forms and Fermat's last theorem (J.Coates and S.-T.Yau eds.), International Press 1995.

[GJ] Godement and H.Jacquet, Zeta functions of simple algebras, LNM 260, Springer 1972.

[Ha] M.Harris, On the local Langlands correspondence, these proceedings.

[He] G.Henniart, Une preuve simple des conjectures de Langlands pour $G L(n)$ sur un corps p-adiques, Invent. Math. 139 (2000), 439-455.

[HT] M.Harris and R.Taylor, The geometry and cohomology of some simple Shimura varieties, PUP 2001.

[Ih] Y.Ihara, On modular curves over finite fields, in "Proceedings of an international colloquium on discrete subgroups of Lie groups and applications to moduli" OUP 1975.

[Il] L.Illusie, Cohomologie de de Rham et cohomologie étale p-adique, 
Astérisque 189-190 (1990), 325-374.

$[\mathrm{J}] \quad$ H.Jacquet, Principal L-functions of the linear group, in "Automorphic forms, representations and $L$-functions 2" AMS 1979.

[JL] H.Jacquet and R.Langlands, Automorphic forms on GL(2), LNM 114, Springer 1970.

[JPSS] H.Jacquet, I.Piatetski-Shapiro and J.Shalika, Automorphic forms on $G L(3)$, Annals of math. 109 (1979), 169-258.

[JS] H.Jacquet and J.Shalika, On Euler products and the classification of automorphic representations II, Amer. J. Math. 103 (1981), 777-815.

[Kol] V.Kolyvagin, On the Mordell-Weil group and the Shafarevich-Tate group of modular elliptic curves, in "Proceedings of the Kyoto International Congress of Mathematicians" Math. Soc. Japan 1991.

[Kot] R.Kottwitz, On the $\lambda$-adic representations associated to some simple Shimura varieties, Invent. Math. 108 (1992), 653-665.

[Laf] L.Lafforgue, Drinfeld varieties and the Langlands program, these proceedings.

[Lan1] R.P.Langlands, Automorphic representations, Shimura varieties and motives. Ein Märchen. in "Automorphic forms, representations and $L$ functions II" AMS 1979.

[Lan2] R.P. Langlands, Base change for $G L(2)$, Annals of Math. Studies 96, Princeton Univ. Press, Princeton, 1980.

[Ma] W.R.Mann, Local level-raising for $G L_{n}$, preprint.

[MB] L.Moret-Bailly, Groupes de Picard et problèmes de Skolem II, Ann. Sci. ENS 22 (1989), 181-194.

[Mi] T.Miyake, Modular forms, Springer 1989.

[P] F.Pop, Embedding problems over large fields, Annals of math 144 (1996), $1-34$.

[R] K.Ribet, Congruence relations between modular forms, in "Proceedings of the Warsaw ICM" Polish Scientific Publishers 1984.

[Sa] D.Savitt, modularity of some potentially Barsotti-Tate Galois representations, preprint.

[Se] J.-P.Serre, Abelian l-adic representations and elliptic curves, Benjamin 1968.

[SW1] C.Skinner and A.Wiles, Residually reducible representations and modular forms, Inst. Hautes Etudes Sci. Publ. Math. 89 (1999), 5-126.

[SW2] C.Skinner and A.Wiles, Base change and a problem of Serre, Duke Math. J. 107 (2001), 15-25.

[SW3] C.Skinner and A.Wiles, Nearly ordinary deformations of irreducible residual representations, Annales de la Faculté de Sciences de Toulouse X (2001), 185-215.

[Tat] J.Tate, Number theoretic background, in A.Borel and W.Casselman "Automorphic forms, representations and $L$-functions", Proc. Symposia in Pure Math. 33 (2), AMS 1979.

[Tay] R.Taylor, On the meromorphic continuation of degree two L-functions, preprint available at http://www.math.harvard.edu/ ${ }^{\sim}$ rtaylor. 
[Tu] J. Tunnell, Artin's conjecture for representations of octahedral type, Bull. AMS 5 (1981), 173-175.

[TW] R.Taylor and A.Wiles, Ring theoretic properties of certain Hecke algebras, Annals of math. 141 (1995), 553-572.

[Wa] N.Wallach, Representations of reductive Lie groups, in "Automorphic forms, representations and $L$-functions I" AMS 1979.

[We] A.Weil, Über die Bestimmung Dirichletscher Reihen durch Funktionalgleichungen, Math. Ann. 168 (1967), 149-156.

[Wi] A.Wiles, Modular elliptic curves and Fermat's last theorem, Annals of math. 141 (1995), 443-551. 